\title{
Intra-arterial hepatic fotemustine for the treatment of liver metastases from uveal melanoma: experience in 101 patients
}

\author{
S. Peters ${ }^{1 \dagger}$, V. Voelter ${ }^{1 \dagger}$, L. Zografos ${ }^{2}$, S. Pampallona ${ }^{3}$, R. Popescu ${ }^{1 \ddagger}$, M. Gillet ${ }^{4}$, W. Bosshard ${ }^{1}$, \\ G. Fiorentini ${ }^{5}$, M. Lotem ${ }^{6}$, R. Weitzen ${ }^{7}$, U. Keilholz ${ }^{8}$, Y. Humblet $^{9}$, S. Piperno-Neumann ${ }^{10}$, \\ R. Stupp ${ }^{1} \&$ S. Leyvraz ${ }^{1 *}$ \\ ${ }^{1}$ Centre Pluridisciplinaire d'Oncologie and ${ }^{4}$ Département de Chirurgie, University of Lausanne Hospitals (CHUV), Lausanne, Switzerland; ${ }^{2}$ Hôpital Ophtalmique Jules \\ Gonin, Lausanne, Switzerland; ${ }^{3}$ ForMed, Evolène, Switzerland; ${ }^{5}$ Ospedale Generale San Giuseppe, Empoli, Italy; ${ }^{6}$ Sharett Institute of Oncology, Hadassah Hebrew \\ University Hospital, Jerusalem, Israel; ${ }^{7}$ Sheba Medical Center, Tel Hashomer, Israel; ${ }^{8}$ Medizinische Klinik II, Benjamin Franklin Hospital of the Free University, Berlin, \\ Germany; ${ }^{9}$ Centre du Cancer, St Luc University Hospital, Brussels, Belgium; ${ }^{10}$ Institut Curie, Paris, France
}

Received 30 October 2005; revised 30 December 2005; accepted 9 January 2006

Background: Exclusive liver metastases occur in up to $40 \%$ of patients with uveal melanoma associated with a median survival of 2-7 months. Single agent response rates with commonly available chemotherapy are below $10 \%$. We have investigated the use of fotemustine via direct intra-arterial hepatic (i.a.h.) administration in patients with uveal melanoma metastases.

Patients and methods: A total of 101 patients from seven centers were treated with i.a.h. fotemustine, administered intra-arterially weekly for a 4-week induction period, and then as a maintenance treatment every 3 weeks until disease progression, unacceptable toxicity or patient refusal.

Results: A median of eight fotemustine infusions per patient were delivered (range 1-26). Catheter related complications occurred in $23 \%$ of patients; however, this required treatment discontinuation in only $10 \%$ of the patients. The overall response rate was 36\% with a median overall survival of 15 months and a 2-year survival rate of 29\%. LDH, time between diagnosis and treatment start and gender were significant predictors of survival.

Conclusions: Locoregional treatment with fotemustine is well tolerated and seems to improve outcome of this poor prognosis patient population. Median survival rates are among the longest reported and one-third of the patients are still alive at 2 years.

Key words: fotemustine, intra-arterial hepatic chemotherapy, uveal melanoma

\section{introduction}

Uveal melanoma is a rare disease, accounting for approximately $0.1 \%$ of all cancer deaths [1]. Its incidence is estimated at 0.6 per 100000 persons/year and seems to remain stable over time, while for cutaneous melanoma a five- to six-fold increase in incidence has been observed [2, 3]. Uveal melanoma differs from its cutaneous counterpart in terms of metastatic pattern and prognosis. Muco-cutaneous melanoma basically spreads via lymphatic vessels often giving rise to multiple locoregional recurrences before systemic disease occurs. In contrast, uveal melanoma metastasizes hematogenously and the liver is the

${ }^{\star}$ Correspondence to: Dr S. Leyvraz, Multidisciplinary Oncology Center, University of Lausanne Hospitals (CHUV), 46, rue du Bugnon, 1011 Lausanne, Switzerland.

Tel: +41-21-314-0149; Fax: +41-21-314-0181; E-mail: Serge.Leyvraz@hospvd.ch

†These authors contributed equally to this work.

*Current affiliation: Oncology Center, Hirslanden Clinic, Aarav, Switzerland. primary and, in up to $90 \%$ of patients, sole metastatic site $[4,5]$. This specific oculo-hepatic tropism remains yet unexplained. Depending on tumor size, the risk of recurrence and metastatic spread is considerable, and the 5-year overall mortality rates range from $20 \%$ for small primary tumors to more than $50 \%$ for larger tumors [6]. In metastatic patients, reported median survival times vary between 2 and 7 months and only 15\% of patients are alive after more than 1 year [7-9].

Metastases from uveal melanoma are considered resistant to commonly available systemic chemo- or chemoimmunotherapy with single agent response rates below 10\%. Most therapies are derived from the experience in cutaneous melanoma. Agents such as dacarbazine (DTIC), cisplatin, interferon and interleukin-2 (IL-2) have been evaluated. In the 1990s fotemustine, a novel nitrosourea with activity against malignant melanoma has been introduced in the clinic [10]. This alkylating agent has a short half-life and a high first-pass liver extraction leading to hepatic concentrations of eight to 47 times higher 
than in normal tissue $[11,12]$. We have investigated the use of fotemustine as a locoregional treatment via direct intra-arterial hepatic administration in patients with metastases from uveal melanoma. In a previous report, we demonstrated the feasibility and good tolerance of this approach, as well as an encouraging response rate of $40 \%$ and a 2 -year survival rate of $23 \%$ [13].

We report here on an extended and multicenter experience of 101 patients who were treated with intra-arterial hepatic (i.a.h.) fotemustine.

\section{patients and methods}

\section{patient selection}

We report on a series of 101 consecutive patients treated between 1990 and 2004 with i.a.h. fotemustine. Data was extracted retrospectively from both prospective clinical databases and from the medical charts of the following institutions: University of Lausanne Hospitals, Switzerland ( $n=66$; including 31 patients previously reported [13]), Institut Curie Paris, France ( $n=19)$, Ospedale Generale San Guiseppe of Empoli, Italy $(n=5)$, Benjamin Franklin Hospital of the Free University of Berlin, Germany ( $n=3)$, St Luc University Hospital of Brussels, Belgium $(n=2)$ and the University Hospitals of Tel Aviv and Jerusalem, Israel $(n=6)$.

Patients were initially enrolled into a prospective phase II feasibility trial. The protocol was approved by the ethical committee. Based on this favorable experience, all subsequent patients were treated according to this protocol. All patients gave informed consent.

Principal eligibility criteria included a performance status of $\leq 2$, histologically confirmed uveal melanoma, liver metastases, the absence of significant tumor bulk outside the liver, normal blood counts and an adequate renal function. Impaired liver function was not an exclusion criteria provided this was due to tumor involvement. Patients had to be ambulatory and fit to undergo surgery for catheter placement. Patients had not received prior chemotherapy.

\section{chemotherapy schedule and drug administration}

Fotemustine (Muphoran ${ }^{\circledR}$ ) was supplied by Servier, Paris, France, as a freeze-dried sterile powder in vials containing $200 \mathrm{mg}$ of active substance diluted with $4 \mathrm{ml}$ ethanol solvent. Before infusion, the drug was further diluted in $250 \mathrm{ml}$ of $5 \%$ injectable glucose solution and protected from light. Fotemustine $100 \mathrm{mg} / \mathrm{m}^{2}$ was administered intra-arterially via the previously placed catheter as a 4-h infusion. It was delivered weekly for a 4-week induction period, followed by a 5 -week rest and then as a maintenance treatment every 3 weeks until disease progression, unacceptable toxicity or patient refusal. In 22 patients, temozolomide at $100 \mathrm{mg} / \mathrm{m}^{2}$ was given for 2 days before the administration of fotemustine during the maintenance phase aiming at methyl guanine methyl transferase (MGMT) depletion $[14,15]$. Antiemetic treatment was administered prophylactically using ondansentron and methylprednisolone.

An implantable catheter connected to a subcutaneous access chamber (Port-A-Cath) was surgically placed into the hepatic artery through the gastroduodenal artery. Debulking surgery was performed whenever feasible.

\section{follow-up visits and assessment of catheter related complications}

Clinical visits were performed weekly during the induction period and every 3 weeks during maintenance treatment and included a complete physical examination, a full blood count and blood chemistry including serum liver function tests. If hematotoxicity grade 3 or 4 was observed, full blood counts were monitored weekly or biweekly until resolution to a grade $\leq 2$. LDH and alkaline phosphatase (AP) levels were recorded at baseline. Catheter-related complications were assessed during the whole treatment period.
Toxicity was graded according to the International Common Toxicity Criteria (NCI, NIH, version 2.0, March 1998).

\section{response evaluation}

Pretreatment tumor assessment, as well as response evaluation, was done using computed tomography (CT) of the chest and abdomen. Response evaluation was performed according to World Health Organization (WHO) criteria [16] at the end of the 5-week rest period after induction and subsequently after every 3 cycles during the maintenance phase. Complete response (CR) was defined as the disappearance of all liver metastases, partial response (PR) as a reduction of $\geq 50 \%$ in the sum of the products of perpendicular diameters of all measurable metastases, and stable disease (SD) as a reduction of $\leq 50 \%$ or an increase of $<25 \%$. Progressive disease (PD) was defined as an increase of $\geq 25 \%$ in the sum of the products of perpendicular diameters of all measurable metastases [17]. Response rate was calculated as the proportion of patients presenting a CR or PR. For the 12 patients with extrahepatic metastases, no increase of pre-existing lesions nor the occurrence of new extrahepatic metastases was allowed in order to be considered as an objective response or stable disease.

\section{statistical considerations}

The primary end point of this study was overall survival (OS), which was defined as time from diagnosis of liver metastases to death. Observation time has been censored at the date of last contact for patients still alive, all survival data was updated in Spring 2005. Statistical analyses were carried out by means of the software package SPSS [18]. Survival percentages over time have been calculated by the Kaplan-Meier method [19] and their corresponding standard errors (SE) with Greenwood's formula [20]. For univariate analyses of OS the $P$ values from the log-rank test [21] are reported for each comparison considered. Estimated hazard ratios (HR) of death, with respect to the indicated reference group, their $95 \%$ confidence intervals $(\mathrm{CI})$ and $P$ values were calculated with (univariate and multivariate) proportional-hazard-regression model [22] with appropriate binary variables to identify each group of interest. The variables considered were (the first category is the reference one for the HR): age ( $\leq 60$ versus $>60$ years versus unknown); performance status ( 0 versus $\geq 1$ versus unknown); gender; center (Lausanne versus others); liver resection (yes versus no versus unknown); $\mathrm{LDH}$ level [normal versus $>1.5 \times$ upper limit of normal (ULN) versus unknown]; alkaline phosphatase (AP) level (normal versus abnormal versus unknown); year of diagnosis of ocular melanoma ( $\leq 1989$ versus $1990-99$ versus $\geq 2000$ ); extrahepatic metastases (yes versus no); time from diagnosis of primary tumor to diagnosis of liver metastases ( $\leq 1$ versus $1-3$ versus $>3$ years); and time from diagnosis of metastases to treatment start ( $\leq 3$ versus $3-6$ versus $>6$ months). Backward elimination ( $P$ value to enter $0.05, P$ value to leave $P=0.10$ ) was used to select the final model. Values of HR greater than unity indicate increased rates of death with respect to the chosen reference category. All probability values are for two-sided tests.

\section{results}

\section{patient and tumor characteristics}

A total of 101 patients from seven centers were included in this analysis. Patients treated at the University Hospital of Lausanne accounted for $65 \%$ of the patients. Patient characteristics are summarized in Table 1; in an intent-to-treat analysis we did not exclude patients with only incomplete information available. The male:female ratio was 1:1 and the median age at the time of diagnosis of liver metastases was 55 years (range 24-74). The majority of patients had a good performance status at the start of treatment with a median Eastern Cooperative Oncology 
Table 1. Patient characteristics

\begin{tabular}{|c|c|}
\hline Characteristic & No. of patients \\
\hline Total & 101 \\
\hline \multicolumn{2}{|l|}{ Gender } \\
\hline Male & 50 \\
\hline Female & 51 \\
\hline \multicolumn{2}{|l|}{ Age (years) } \\
\hline \multicolumn{2}{|c|}{ Median 55 (range 24-74) } \\
\hline $24-60$ & 59 \\
\hline$>60$ & 36 \\
\hline Unknown & 6 \\
\hline \multicolumn{2}{|l|}{ PS at diagnosis } \\
\hline \multicolumn{2}{|c|}{ Median 0 (range $0-2$ ) } \\
\hline 0 & 64 \\
\hline $1+2$ & 28 \\
\hline Unknown & 9 \\
\hline \multicolumn{2}{|c|}{ Extrahepatic metastases } \\
\hline Yes & 12 \\
\hline No & 89 \\
\hline \multicolumn{2}{|l|}{ Liver resection } \\
\hline Yes & 39 \\
\hline No & 51 \\
\hline Unknown & 11 \\
\hline \multicolumn{2}{|c|}{ Elevated LDH at diagnosis } \\
\hline Yes & 21 \\
\hline No & 66 \\
\hline Unknown & 14 \\
\hline \multicolumn{2}{|c|}{ Abnormal alk. phosphatase at diagnosis } \\
\hline Yes & 31 \\
\hline No & 41 \\
\hline Unknown & 29 \\
\hline \multicolumn{2}{|c|}{$\begin{array}{l}\text { Time interval from diagnosis of ocular melanoma to } \\
\text { hepatic metastases }\end{array}$} \\
\hline \multicolumn{2}{|c|}{ Median 34 months (range 3-332) } \\
\hline$<1$ year & 11 \\
\hline$>1-3$ years & 42 \\
\hline$>3-28$ years & 48 \\
\hline \multicolumn{2}{|c|}{$\begin{array}{l}\text { Time interval from diagnosis of liver metastases to } \\
\text { treatment start }\end{array}$} \\
\hline \multicolumn{2}{|c|}{ Median 1.9 months (range $0.1-45$ ) } \\
\hline$<3$ months & 77 \\
\hline$>3-6$ months & 18 \\
\hline$>6$ months & 6 \\
\hline
\end{tabular}

PS, performance status.

Group (ECOG) stage of 0 (range 0-2) [17]. The liver was the only site of metastases in 89 patients and the liver metastases were histologically confirmed in the great majority of patients. Twelve patients also had extrahepatic disease including skin, bone, lung or peritoneal metastases, but were included because liver was the main site of disease and the location determining survival. Thirty-eight patients underwent debulking (nonradical) surgery of liver metastases (metastasectomy, segmentectomy, right or left hepatectomy) at the time of catheter placement. Elevated baseline LDH levels were observed in 21 patients and AP levels were abnormal in 31. The time from diagnosis of the primary tumor to the diagnosis of liver metastases was highly variable (median 34 months, range 3-332 months) with almost half of the patients developing metastatic disease after 3 years, and in one patient distant spread occurred as late as 28 years. The median time from diagnosis of liver metastases to first administration of fotemustine was 1.9 months (range 0.1-45 months). Six patients had been referred to the medical oncologist later than 6 months after diagnosis of hepatic metastases.

\section{treatment delivery and complications}

Overall, 918 infusions of fotemustine with a median of eight per patient were delivered (range 1-26). The majority of the patients (77\%) received all four administrations of fotemustine during the induction period (median four cycles, range $0-4)$. The median number of maintenance cycles administered was five (range 0-22). Seventeen patients did not proceed to maintenance phase because of toxicity $(n=3)$, disease progression $(n=10)$, one patient refused to continue chemotherapy and for three patients no information was available. Eighty-four patients started maintenance therapy and received a median of five additional cycles (range 1-22). Eighteen patients received fotemustine for more than 6 months (up to 25 months), with low toxicity throughout the treatment duration.

Severe grade $3 / 4$ toxicity was observed in $11 \%$ of patients, mainly hematotoxicity (4\%). In addition, grade 2 hematotoxicity, and in particular thrombocytopenia, occurred in $11 \%$ of patients.

Placement of an intra-arterial catheter is associated with a risk of catheter thrombosis, dislocation and, to a lesser extent, catheter stenosis/obstruction or leakage. Overall, complications related to the catheter, namely thrombosis, occurred in 21 patients, usually only after several months of treatment. More than three-quarters of the patients with a catheter thrombosis could nevertheless complete the chemotherapy as planned. Early treatment discontinuation due to the catheter was necessary in nine patients after a median treatment duration of 4 months. Two of them presented catheter thrombosis, two of them catheter stenosis/obstruction and two of them dislocation. Organ damage in the form of pancreatitis and sclerosing cholangitis developed in two patients. One patient could not start chemotherapy because of the appearance of a postoperative icterus, following the surgical procedure in the context of extensive liver disease and hepatic insufficiency.

\section{therapeutic activity}

Of the 101 patients, 21 achieved a partial response and 15 patients had a radiological complete response, for an overall response rate of $36 \%$. In 48 patients the disease was stable, for a median duration of 9.4 months. Seventeen per cent of the patients had progressive disease at first radiological assessment.

Median overall survival for the whole group was 15 months (95\% confidence interval (CI) 12.1-17.6 months) with a 1 -year survival rate of $67 \%$ (95\% CI $57-75 \%)$. Twenty-nine per cent of the patients were still alive at 2 years and $12 \%$ at 3 years (Figure 1 ).

Multivariate and univariate analysis did not show any impact of age, performance status, the presence of extrahepatic disease at baseline, or the interval between diagnosis of primary 


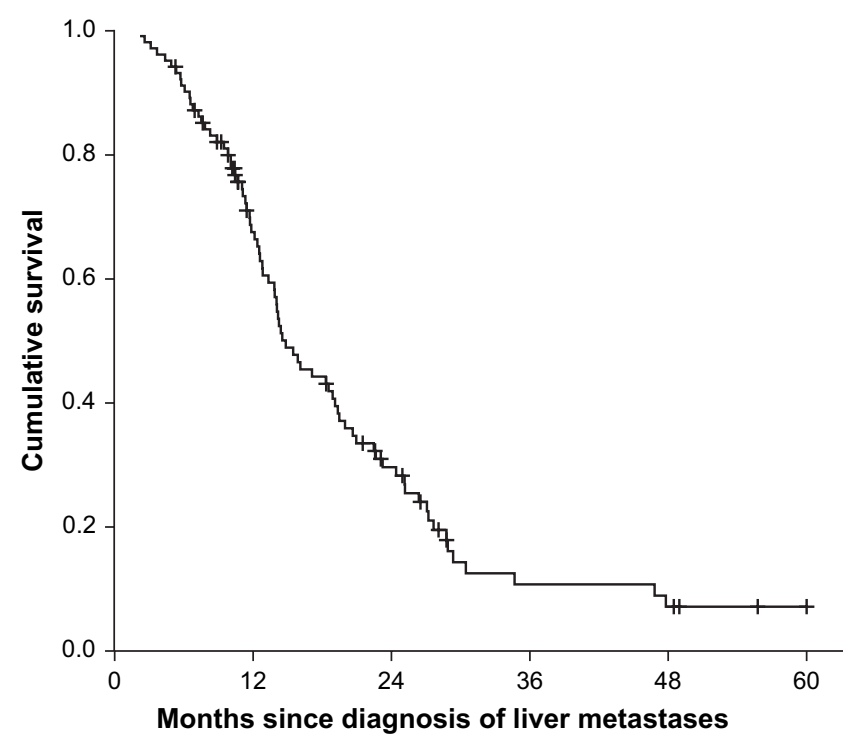

Figure 1. Overall survival of patients since diagnosis of liver metastasis. Survival percentages are shown over time in months. Percentages have been calculated according the Kaplan-Meier method. Median overall survival for the whole group was 15 months (95\% CI 12.1-17.6). Curves are truncated at 5 years (numbers show censored observations).

tumor and liver metastases on outcome. After scrutiny of all variables according to a forward selection approach, $\mathrm{LDH}$, time between diagnosis of liver metastases and treatment start, resection of liver metastases as well as gender were significant predictors of survival (Table 2). Elevated LDH levels were the strongest predictors for survival with a median survival time of 9.5 months compared with 19.4 months with levels $\leq 1.5 \times$ ULN (multivariate HR $=3.7,95 \%$ CI 2.0-7.0, $P<0.001)$ and 15.5 months for patients with unknown levels (multivariate $\mathrm{HR}=0.9,95 \% \mathrm{CI} 0.4-1.8, P<0.710$ ). Male gender was an unfavorable prognostic factor for survival in this series (multivariate $\mathrm{HR}=1.8,95 \%$ CI 1.1-3.0, $P=0.026$ ). Resection of liver metastases was performed in 39 patients. The median survival of these patients was 25 months (95\% CI 19.8-30.5) compared with 13 months (95\% CI 10.0-15.6) for those who did not have resection and 13.4 months (95\% CI 11.0-15.7) for patients with unknown status $(P=0.001)$. In multivariate analysis patients with resection showed a better though not significant survival; significance was observed for patients with unknown status. The interval between diagnosis of liver metastases and the time of chemotherapy start was shown to be related to patients' survival. Patients who started chemotherapy later than 6 months after diagnosis incurred a reduced risk of death; however, this was of borderline significance.

\section{discussion}

We report here on an extended experience of intra-arterial intrahepatic chemotherapy with fotemustine in patients with metastatic uveal melanoma to the liver. To our knowledge this is the largest series of homogeneously treated patients with this rare disease expanding on our previously reported local experience [13]. The study population is representative of the
Table 2. Factors influencing survival by multivariate Cox regression with backward elimination

\begin{tabular}{|c|c|c|c|}
\hline & HR & 95\% CI for HR & $P$ value \\
\hline \multicolumn{4}{|l|}{$\mathrm{LDH}>1.5 \mathrm{UNL}$} \\
\hline No & 1 & Reference & \\
\hline Yes & 3.7 & $2.0-7.0$ & $<0.001$ \\
\hline Unknown & 0.9 & $0.4-1.8$ & 0.710 \\
\hline \multicolumn{4}{|l|}{ Liver resection } \\
\hline No & 1 & Reference & \\
\hline Yes & 0.7 & $0.4-1.3$ & 0.253 \\
\hline Unknown & 2.4 & $1.1-4.9$ & 0.021 \\
\hline \multicolumn{4}{|l|}{ Gender } \\
\hline Female & 1 & Reference & \\
\hline Male & 1.8 & $1.1-3.0$ & 0.026 \\
\hline \multicolumn{4}{|c|}{$\begin{array}{l}\text { Months from liver metastasis to } \\
\text { chemotherapy }\end{array}$} \\
\hline$<3$ months & 1 & Reference & \\
\hline$>3-6$ months & 1.5 & $0.8-2.8$ & 0.229 \\
\hline$>6$ months & 0.3 & $0.1-1.1$ & 0.067 \\
\hline
\end{tabular}

HR, hazard ratios of death; CI, confidence intervals.

Estimated hazard ratios (HR) of death are calculated with respect to the indicated reference group.

type of patient presenting with metastatic uveal melanoma. Almost $90 \%$ of patients presented with liver involvement as a single metastatic site at diagnosis, in line with the particular oculo-hepatic tropism of uveal melanoma cells. Hence, it is the metastatic liver involvement that accounts for the poor outcome of these patients with median survival rates that do not exceed a year in historical series. Interestingly, patients presenting only extrahepatic metastasis have a better prognosis [7-9].

There have been few treatment options for this patient population in the past. Most systemic chemo-/immunotherapy regimens, similar to cutaneous melanoma, demonstrated even less activity in uveal melanoma [23]. Both tumor entities differ considerably in their molecular profile and biological behavior despite their common embryological origin [24-26]. Uveal melanoma represents a distinct entity. Patients with metastatic uveal melanoma should be considered for specific treatment protocols, rather than being included in trials for metastatic skin melanoma.

Since the liver represents the critical organ determining patients' outcome, locoregional treatment regimens have been developed in the past. Fotemustine is a third-generation nitrosourea with a high hepatic extraction. In early clinical trials it has shown promising activity in uveal melanoma [13, 27-30]. Furthermore, this prolonged arterial intrahepatic administration over $4 \mathrm{~h}$ reduced the plasma AUC by $50 \%$, translating into decreased systemic toxicity compared with the intravenous schedule [11].

The previously reported experience in a single center phase II trial on 30 patients [13] has been confirmed in the present analysis on 101 patients treated in different institutions. We observed a $36 \%$ response rate (including 15\% complete responses), which reflects the excellent locoregional activity of fotemustine. This translates into a median overall survival of 15 months with almost one-third of the patients still alive at 
2 years. These results compare favorably with other locoregional treatment options such as arterial chemoembolization or isolated hepatic perfusion [31-33]. The latter techniques are associated with substantial morbidity and mortality, necessitating prolonged hospitalization. Locoregional chemotherapy with fotemustine can be delivered on an outpatient basis, improves local control and has a favorable systemic toxicity profile.

The need for catheter placement and associated complications is a major obstacle to all locoregional treatments (i.e. arterial chemoembolization, isolated hepatic perfusion) [27, 32-34]. Although in the present series, almost one-quarter of the patients experienced a complication due to the arterial catheter, these complications required treatment discontinuation in only nine patients. Our experience is in agreement with reports of intra-arterial chemotherapy for liver metastases of colorectal cancer. In these reports, $14 \%$ of patients experienced catheterrelated complications rendering the infusional device unusable, and were mostly thrombosis and catheter dislocation $[35,36]$.

Hematotoxicity reported in this series is lower compared with reports of intravenous fotemustine administration. This is possibly due to the first pass effect and hepatic extraction. However, due in part to the retrospective data collection, there may be an underestimation of the toxicity.

Retrospective series attempted to define prognostic factors for patients with uveal melanoma. The strongest predictive factors for metastatic spread are macroscopic and histopathologic findings of the primary tumor [37-39]. Features like invasion of the ciliary body, the sclera or the anterior equator of the eye, tumor diameter as well as epitheloid cells, monosomy of chromosome 3 [40] and patterns of vascular mimicry [41] have been uniformly associated with increased risk for distant metastases. However, the determination of prognostic factors in the metastatic setting is less clear and controversial results have been reported on factors like age, gender, debulking surgery or biochemical parameters (serum alkaline phosphatase, $\gamma \mathrm{GT}$ and LDH) [42-44]. Our present data confirm elevated $\mathrm{LDH}$ to be a significant negative prognostic factor. Although the influence of resection of liver metastases has never been studied within a randomized trial, previous reports suggest that metastasectomy or surgical tumor reduction showed no prognostic significance. Only macroscopically complete tumor resection seemed to improve patients' survival [5]. In our study, in multivariate analysis, patients with tumor resection showed a better, though not statistically significant, survival. This may reflect complete tumor resection performed in some of the operated patients, but also patient selection of the fittest for surgery. How far disease evolution is different between men and women remains controversial. In our series, male gender has been shown to be a negative prognostic factor for survival. A long interval between the time of diagnosis of liver metastases and treatment start had a favorable prognostic impact. Interpretation of this finding should be handled with caution due to the small group of patients (six). It might represent a selection of patients with a favorable natural history of metastatic ocular melanoma. However, their exclusion from statistical analysis does not modify overall survival.

In summary, we confirmed in an extended patient population that intra-arterial, intrahepatic fotemustine is an effective and well-tolerated treatment against liver metastases from uveal melanoma. Median survival rates are among the longest reported and one-third of the patients were still alive at 2 years. Compared with historical series and systemic treatment approaches, locoregional treatment with fotemustine seems to improve outcome of this poor prognosis patient population [28, 45-47]. However, although a non-randomized comparative trial between intra-arterial hepatic and intravenous administration of fotemustine could demonstrate a higher response rate with the locoregional approach, it did not translate into a significant benefit in overall survival [28]. Improved outcomes with recent systemic treatments may also reflect patient selection and earlier detection of metastasis (lead time bias) with median survival rates comparable to our series even in the absence of a objective antitumor response $[45,48]$. This underlines the importance of prospective controlled trials before embracing a new treatment approach. The melanoma group of the European Organisation for Research and Treatment of Cancer (EORTC) recently opened a large phase III trial (EORTC 18021), comparing intra-arterial hepatic fotemustine administration with intravenous systemic fotemustine. The primary end point of this trial is overall survival [49].

\section{references}

1. Hakulinen $T$, Teppo $L$, Saxen E. Cancer of the eye, a review of trends and differentials. World Health Stat Q 1978; 31: 143-158.

2. Osterlind $A$. Trends in incidence of ocular malignant melanoma in Denmark 1943-1982. Int J Cancer 1987; 40: 161-164.

3. Scotto J, Fraumeni JF Jr, Lee JA. Melanomas of the eye and other noncutaneous sites: epidemiologic aspects. J Natl Cancer Inst 1976; 56: 489-491.

4. Assessment of metastatic disease status at death in 435 patients with large choroidal melanoma in the Collaborative Ocular Melanoma Study (COMS): COMS report no. 15. Arch Ophthalmol 2001; 119: 670-676.

5. Desjardins L, Dorval T, Levy C et al. Etude randomisée de chimiothérapie adjuvante par le Déticène dans le mélanome choroïdien. Ophthalmologie 1998; 12: $168-173$

6. Diener-West M, Hawkins BS, Markowitz JA, Schachat AP. A review of mortality from choroidal melanoma. II. A meta-analysis of 5-year mortality rates following enucleation, 1966 through 1988. Arch Ophthalmol 1992; 110: 245-250.

7. Bedikian AY, Kantarjian H, Young SE, Bodey GP. Prognosis in metastatic choroidal melanoma. South Med J 1981; 74: 574-577.

8. Kath R, Hayungs J, Bornfeld $\mathrm{N}$ et al. Prognosis and treatment of disseminated uveal melanoma. Cancer 1993; 72: 2219-2223.

9. Gragoudas ES, Egan KM, Seddon JM et al. Survival of patients with metastases from uveal melanoma. Ophthalmology 1991; 98: 383-390.

10. Avril MF, Aamdal S, Grob JJ et al. Fotemustine compared with dacarbazine in patients with disseminated malignant melanoma: a phase III study. J Clin Oncol 2004; 22: 1118-1125.

11. Fety $R$, Lucas $C$, Solere $P$ et al. Hepatic intra-arterial infusion of fotemustine: pharmacokinetics. Cancer Chemother Pharmacol 1992; 31: 118-122.

12. Collins JM. Pharmacologic rationale for hepatic arterial therapy. Recent Results Cancer Res 1986; 100: 140-147.

13. Leyvraz S, Spataro V, Bauer J et al. Treatment of ocular melanoma metastatic to the liver by hepatic arterial chemotherapy. J Clin Oncol 1997; 15: 2589-2595.

14. Gerson SL. Clinical relevance of MGMT in the treatment of cancer. J Clin Oncol 2002; 20: 2388-2399.

15. Gander M, Leyvraz S, Decosterd $L$ et al. Sequential administration of temozolomide and fotemustine: depletion of 06-alkyl guanine-DNA transferase in blood lymphocytes and in tumours. Ann Oncol 1999; 10: 831-838. 
16. World Health Organization. WHO Handbook for Reporting Results of Cancer Treatment. Geneva: WHO 1979.

17. Oken MM, Creech RH, Tormey DC et al. Toxicity and response criteria of the Eastern Cooperative Oncology Group. Am J Clin Oncol 1982; 5: 649-655.

18. SPSS. Statistical Package for the Social Sciences. Release 13 Chicago, Illinois.

19. Kaplan E, Meier P. Nonparametric estimation from incomplete observations. J Am Stat Assoc 1958; 53: 457-481.

20. Greenwood M. Reports on Public Health and Medical Subjects: The Natural Duration of Cancer. HSMO 1926; 33: 1-16.

21. Peto R, Pike MC, Armitage $P$ et al. Design and analysis of randomized clinical trials requiring prolonged observation of each patient. II. analysis and examples. Br J Cancer 1977; 35: 1-39.

22. Cox D. Regression models and life tables. J R Stat Soc B 1972; 34: 187-220.

23. Bedikian AY, Legha SS, Mavligit G et al. Treatment of uveal melanoma metastatic to the liver: a review of the M. D. Anderson Cancer Center experience and prognostic factors. Cancer 1995; 76: 1665-1670.

24. Rimoldi D, Salvi S, Lienard D et al. Lack of BRAF mutations in uveal melanoma. Cancer Res 2003; 63: 5712-5715.

25. Onken MD, Worley LA, Ehlers JP, Harbour JW. Gene expression profiling in uveal melanoma reveals two molecular classes and predicts metastatic death. Cancer Res 2004; 64: 7205-7209.

26. Cree IA. Cell cycle and melanoma-two different tumours from the same cell type. J Pathol 2000; 191: 112-114.

27. Egerer G, Lehnert T, Max R et al. Pilot study of hepatic intraarterial fotemustine chemotherapy for liver metastases from uveal melanoma: a single-center experience with seven patients. Int J Clin Oncol 2001; 6: 25-28.

28. Becker JC, Terheyden P, Kampgen $E$ et al. Treatment of disseminated ocular melanoma with sequential fotemustine, interferon alpha, and interleukin 2. Br J Cancer 2002; 87: 840-845.

29. Jacquillat $C$, Khayat $D$, Banzet $P$ et al. Final report of the French multicenter phase II study of the nitrosourea fotemustine in 153 evaluable patients with disseminated malignant melanoma including patients with cerebral metastases. Cancer 1990; 66: 1873-1878.

30. Khayat D, Cour V, Bizzari JP et al. Fotemustine (S 10036) in the intra-arterial treatment of liver metastasis from malignant melanoma. A phase II Study. Am J Clin Oncol 1991; 14: 400-404.

31. Cantore M, Fiorentini G, Aitini E et al. Intra-arterial hepatic carboplatin-based chemotherapy for ocular melanoma metastatic to the liver. Report of a phase II study. Tumori 1994; 80: 37-39.

32. Feun LG, Reddy KR, Yrizarry JM et al. A phase I study of chemoembolization with cisplatin and lipiodol for primary and metastatic liver cancer. Am J Clin Oncol 1994; 17: 405-410.

33. Agarwala SS, Panikkar R, Kirkwood JM. Phase I/II randomized trial of intrahepatic arterial infusion chemotherapy with cisplatin and chemoembolization with cisplatin and polyvinyl sponge in patients with ocular melanoma metastatic to the liver. Melanoma Res 2004; 14: 217-222.
34. Feldman ED, Pingpank JF, Alexander HR Jr. Regional treatment options for patients with ocular melanoma metastatic to the liver. Ann Surg Oncol 2004; 11: 290-297.

35. Lorenz M, Muller HH. Randomized, multicenter trial of fluorouracil plus leucovorin administered either via hepatic arterial or intravenous infusion versus fluorodeoxyuridine administered via hepatic arterial infusion in patients with nonresectable liver metastases from colorectal carcinoma. J Clin Oncol 2000; 18: 243-254.

36. Kemeny N, Huang $\mathrm{Y}$, Cohen AM et al. Hepatic arterial infusion of chemotherapy after resection of hepatic metastases from colorectal cancer. N Engl J Med 1999; 341: 2039-2048.

37. Singh AD, Shields CL, Shields JA. Prognostic factors in uveal melanoma. Melanoma Res 2001; 11: 255-263.

38. Seregard S, Kock E. Prognostic indicators following enucleation for posterior uveal melanoma. A multivariate analysis of long-term survival with minimized loss to follow-up. Acta Ophthalmol Scand 1995; 73: 340-344.

39. McLean IW, Foster WD, Zimmerman LE. Uveal melanoma: location, size, cell type, and enucleation as risk factors in metastasis. Hum Pathol 1982; 13: $123-132$.

40. Prescher G, Bornfeld N, Hirche $\mathrm{H}$ et al. Prognostic implications of monosomy 3 in uveal melanoma. Lancet 1996; 347: 1222-1225.

41. Toivonen P, Makitie T, Kujala E, Kivela T. Microcirculation and tumor-infiltrating macrophages in choroidal and ciliary body melanoma and corresponding metastases. Invest Ophthalmol Vis Sci 2004; 45: 1-6.

42. Eskelin S, Pyrhonen S, Hahka-Kemppinen M et al. A prognostic model and staging for metastatic uveal melanoma. Cancer 2003; 97: 465-475.

43. Salmon RJ, Levy C, Plancher $C$ et al. Treatment of liver metastases from uveal melanoma by combined surgery-chemotherapy. Eur J Surg Oncol 1998; 24: $127-130$.

44. Sirott MN, Bajorin DF, Wong GY et al. Prognostic factors in patients with metastatic malignant melanoma. A multivariate analysis. Cancer 1993; 72: 3091-3098.

45. Kivela T, Suciu S, Hansson J et al. Bleomycin, vincristine, lomustine and dacarbazine (BOLD) in combination with recombinant interferon alpha-2b for metastatic uveal melanoma. Eur J Cancer 2003; 39: 1115-1120.

46. Mavligit GM, Charnsangavej C, Carrasco $\mathrm{CH}$ et al. Regression of ocular melanoma metastatic to the liver after hepatic arterial chemoembolization with cisplatin and polyvinyl sponge. JAMA 1988; 260: 974-976.

47. Schmittel A, Scheulen ME, Bechrakis NE et al. Phase II trial of cisplatin, gemcitabine and treosulfan in patients with metastatic uveal melanoma. Melanoma Res 2005; 15: 205-207.

48. Kodjikian L, Grange JD, Baldo $S$ et al. Prognostic factors of liver metastases from uveal melanoma. Graefes Arch Clin Exp Ophthalmol 2005; 243: 985-993.

49. Intravenous versus intra-arterial fotemustine chemotherapy in patients with liver metastases from uveal melanoma: a randomized phase III study of the EORTC Melanoma Group. EORTC protocol 18021; Study coordinator: Serge.Leyvraz@hospvd.ch. 